\title{
Older patients (aged $\geq 60$ years) with previously untreated advanced-stage classical Hodgkin lymphoma: a detailed analysis from the phase III ECHELON-1 study
}

Haematologica 2022

Volume 107(5):1086-1094

\section{Correspondence:}

ANDREW M. EVENS

ae378@cinj.rutgers.edu

Received: February 1, 2021.

Accepted: June 17, 2021.

Pre-published: June 24, 2021.

https://doi.org/10.3324/haematol.2021.278438

(C)2022 Ferrata Storti Foundation

Material published in Haematologica is covered by copyright. All rights are reserved to the Ferrata Storti Foundation. Use of published material is allowed under the following terms and conditions:

https://creativecommons.org/licenses/by-nc/4.0/legalcode. Copies of published material are allowed for personal or internal use. Sharing published material for non-commercial purposes is subject to the following conditions:

https://creativecommons.org/licenses/by-nc/4.0/legalcode, sect. 3. Reproducing and sharing published material for commercial purposes is not allowed without permission in writing from the publisher.
Andrew M. Evens, ${ }^{1}$ Joseph M. Connors, ${ }^{2}$ Anas Younes,${ }^{3}$ Stephen M. Ansell ${ }^{4}$ Won Seog Kim, ${ }^{5}$ John Radford, ${ }^{6}$ Tatyana Feldman, ${ }^{7}$ Joseph Tuscano, ${ }^{8}$ Kerry J. Savage, ${ }^{2}$ Yasuhiro Oki, ${ }^{9}$ Andrew Grigg, ${ }^{10}$ Christopher Pocock, ${ }^{11}$ Monika Dlugosz-Danecka, ${ }^{12}$ Keenan Fenton, ${ }^{13}$ Andres Forero-Torres, ${ }^{13}$ Rachael Liu, ${ }^{14}$ Hina Jolin, ${ }^{14}$ Ashish Gautam ${ }^{14}$ and Andrea Gallamini ${ }^{15}$

${ }^{1}$ Division of Blood Disorders, Rutgers Cancer Institute of New Jersey, New Brunswick, NJ, USA; ${ }^{2} \mathrm{BC}$ Cancer Center for Lymphoid Cancer and Department of Medical Oncology, Vancouver, BC, Canada; ${ }^{3}$ Memorial Sloan Kettering Cancer Center, New York, NY, USA; ${ }^{4}$ Mayo Clinic, Rochester, NY, USA; ${ }^{5}$ Sungkyunkwan University School of Medicine, Samsung Medical Center, Seoul, South Korea; ${ }^{6}$ University of Manchester and the Christie NHS Foundation Trust Manchester Academic Health Science Center, Manchester, UK; ${ }^{7}$ John Theurer Cancer Center, Hackensack, NJ, USA; ${ }^{8}$ UC Davis Cancer Center, Sacramento, CA, USA; ${ }^{9}$ Genentech, South San Francisco, CA, USA; ${ }^{10}$ Olivia Newton-John Cancer Wellness and Research Center, Austin Health and Department of Clinical Haematology, Austin Hospital, Heidelberg, Australia; ${ }^{11}$ Haematology, East Kent Hospitals, Canterbury, UK; ${ }^{12}$ Maria Sklodowska-Curie National Research Institute of Oncology, Krakow, Poland; ${ }^{13}$ Seagen Inc., Bothell, WA, USA; ${ }^{14}$ Millennium Pharmaceuticals, Inc., Cambridge, MA, USA, a wholly owned subsidiary of Takeda Pharmaceutical Company Limited and ${ }^{15}$ Research and Innovation Department, A. Lacassagne Cancer Center, Nice, France

${ }^{\circ}$ Current affiliation: Haematology (Early and Late Stage) Oncology R\&D, AstraZeneca, New York, NY, USA

\section{ABSTRACT}

$\Gamma$ ffective and tolerable treatments are needed for older patients with classical Hodgkin lymphoma. We report results for older patients with classical Hodgkin lymphoma treated in the large phase III ECHELON-1 study of frontline brentuximab vedotin plus doxorubicin, vinblastine, and dacarbazine $(A+A V D)$ versus doxorubicin, bleomycin, vinblastine, and dacarbazine (ABVD). Modified progression-free survival per independent review facility for older versus younger patients (aged $\geq 60$ vs. $<60$ years) was a pre-specified subgroup analysis; as the ECHELON-1 study was not powered for these analyses, reported $P$-values are descriptive. Of 1,334 enrolled patients, $186(14 \%)$ were aged $\geq 60$ years (A+AVD: $n=84, A B V D: n=102)$; results below refer to this age group. Modified progression-free survival per independent review facility was similar in the two arms at 24 months (A+AVD: 70.3\% [95\% confidence interval (CI): 58.4-79.4], ABVD: 71.4\% [95\% CI: 60.5-79.8], hazard ratio $(\mathrm{HR})=1.00$ [95\% CI: 0.58-1.72], $P=0.993$ ). After a median follow-up of 60.9 months, 5-year progression-free survival per investigator was $67.1 \%$ with $A+A V D$ versus $61.6 \%$ with $A B V D$ ( $\mathrm{HR}=0.820$ [95\% CI: 0.494-1.362], $P=0.443$ ). Comparing $A+A V D$ versus ABVD, grade $3 / 4$ peripheral neuropathy occurred in $18 \%$ versus $3 \%$; any-grade febrile neutropenia in $37 \%$ versus $17 \%$; and any-grade pulmonary toxicity in $2 \%$ versus $13 \%$, respectively, with three $(3 \%)$ pulmonary toxicity-related deaths in patients receiving $A B V D$ (none in those receiving $A+A V D$ ). Altogether, A+AVD showed overall similar efficacy to ABVD with survival rates in both arms comparing favorably to those of prior series in older patients with advanced-stage classical Hodgkin lymphoma. Compared to ABVD, A+AVD was associated with higher rates of neuropathy and neutropenia, but lower rates of pulmonary-related toxicity. Trials registered at ClinicalTrials.gov identifiers: NCT01712490; EudraCT number: 2011-005450-60. 


\section{Introduction}

Older patients (aged $\geq 60$ years) account for approximately $20-25 \%$ of cases of classical Hodgkin lymphoma (cHL) in population-based studies. ${ }^{1-3}$ While outcomes for younger patients with cHL have improved significantly in recent decades, similar progress has not been seen for older patients, ${ }^{4}$ in particular for those with advancedstage disease. ${ }^{2,3,5}$ This has been attributable to biological disease differences and co-morbidities associated with advanced age resulting in poor tolerance of chemotherapy and increased incidence of severe toxicities, including treatment-related deaths. ${ }^{4,6}$ Intensive regimens, such as bleomycin, etoposide, doxorubicin, cyclophosphamide, vincristine, procarbazine, and prednisone (BEACOPP) are too toxic for older patients and may result in increased treatment-related mortality. In addition, bleomycin, a component of the doxorubicin, bleomycin, vinblastine, and dacarbazine (ABVD) regimen is associated with a significantly elevated risk of pulmonary toxicity in older patients, ${ }^{5,8-10}$ particularly in those aged $\geq 70$ years. ${ }^{11-13}$

Brentuximab vedotin has been evaluated as an alternative treatment approach in older, less fit patients with previously untreated cHL, both as monotherapy ${ }^{14,15}$ and in combination regimens. ${ }^{16-18}$ Results from these earlyphase trials demonstrated tolerability and encouraging efficacy, with objective response rates of $98-100 \%$ and complete response rates of $44-87 \% .{ }^{16-18}$ Sequential therapy in a phase II multicenter study with two cycles of brentuximab vedotin followed by six cycles of doxorubicin, vinblastine, and dacarbazine (AVD) yielded encouraging results. ${ }^{19}$

There has been a relative paucity of randomized phase III clinical trials in the frontline cHL setting that have included older patients in the contemporary era. In the primary analysis of the phase III ECHELON-1 study performed after a median follow-up of 24.6 months, frontline administration of brentuximab vedotin in combination with AVD (A+AVD) significantly improved the primary endpoint, modified progression-free survival (PFS) per independent review facility (IRF), compared with ABVD (hazard ratio $[\mathrm{HR}]=0.77$ [95\% confidence interval (CI): $0.60-0.98], P=0.035) .{ }^{20}$ Exploratory 3 - and 5-year analyses reported continued provision of per-investigator PFS benefits for A+AVD compared with ABVD. ${ }^{21,22}$ Here we report the results of pre-specified analyses and post hoc analyses with extended follow-up of the efficacy and safety of $\mathrm{A}+\mathrm{AVD}$ versus $\mathrm{ABVD}$ in 186 older cHL patients (aged $\geq 60$ years).

\section{Methods}

\section{Study design and assessments}

The study design and population of patients for the openlabel, global, randomized, phase III ECHELON-1 study have been described previously. ${ }^{20}$ Briefly, patients aged $\geq 18$ years (no upper age limit) with histologically confirmed, advanced (Ann Arbor stage III//V) cHL who had received no prior systemic chemotherapy or radiotherapy were randomized 1:1 to receive A+AVD (brentuximab vedotin $1.2 \mathrm{mg} / \mathrm{kg}$, doxorubicin 25 $\mathrm{mg} / \mathrm{m}^{2}$, vinblastine $6 \mathrm{mg} / \mathrm{m}^{2}$, and dacarbazine $375 \mathrm{mg} / \mathrm{m}^{2}$ ) or ABVD (doxorubicin $25 \mathrm{mg} / \mathrm{m}^{2}$, bleomycin 10 units $/ \mathrm{m}^{2}$, vinblastine $6 \mathrm{mg} / \mathrm{m}^{2}$, and dacarbazine $\left.375 \mathrm{mg} / \mathrm{m}^{2}\right)$ intravenously on days 1 and 15 of each 28-day cycle for up to six cycles. Dose reductions and modifications for brentuximab vedotin, including for the management of peripheral neuropathy, have been described previously. ${ }^{20}$

Patients were assessed for response to study treatment per IRF in accordance with the 2007 Revised Response Criteria for Malignant Lymphoma. ${ }^{23}$ Computed tomography scans were performed at screening, at the end of cycle 2, after administration of the last dose of frontline therapy, and during follow-up (every 3 months in the first year, and every 6 months thereafter). Positron emission tomography (PET) scans were performed at screening, the end of cycle 2, and the end of treatment. Adverse events were graded according to the National Cancer Institute Common Terminology Criteria for Adverse Events version 4.03.

ECHELON-1 was conducted in accordance with regulatory requirements; the protocol was approved by the institutional review boards and ethics committees at each registered site. Written informed consent, in accordance with local ethics committee instructions, was mandatory before enrollment. This study was conducted according to the guideline of the International Conference on Harmonization Good Clinical Practice.

\section{Endpoints and analyses}

The primary endpoint for ECHELON-1 was modified PFS per IRF, defined as the time to progression, death, or evidence of non-complete response per IRF (Deauville score $\geq 3$ ) after completion of frontline therapy, followed by subsequent anticancer therapy (chemotherapy and/or radiotherapy). Overall survival (OS) was defined as the time from randomization to death from any cause and was the key secondary endpoint.

Here we report a pre-specified subgroup analysis of modified PFS per IRF in older patients (defined as $\geq 60$ years of age), as well as exploratory analyses, including PFS per investigator assessment (the time from randomization to relapse/progression or death) and safety. Subgroups of patients for efficacy and safety analyses were derived from the intention-to-treat (all randomized patients enrolled in ECHELON-1) and safety (all patients who received at least one dose of trial drug) populations, respectively. Following the primary analysis, the protocol did not require investigators to submit further information to the IRF, thus extended follow-up for analysis of modified PFS or PFS by IRF was not conducted. Modified PFS and PFS were summarized using the Kaplan-Meier methodology. ECHELON-1 was not powered for age-based subgroup analyses, so reported $P$-values are descriptive and without multiplicity adjustment.

\section{Results}

\section{Patients}

As reported previously, 1,334 patients were included in the intention-to-treat population; ${ }^{20}$ of whom 186/1,334 (14\%; A+AVD: $n=84, A B V D: n=102)$ were aged $\geq 60$ years $(\mathrm{A}+\mathrm{AVD}$ arm: median age 68 years [range, 60-82], ABVD arm: median age 66 years [range, 60-83]) and were included in these sub-analyses. Patients' demographics and disease characteristics were well balanced across the treatment arms in both older and younger patients. Within both arms, older patients tended to have a poorer Eastern Cooperative Oncology performance status than younger patients (Table 1).

\section{Efficacy in older patients}

At the time of the primary analysis the median followup for older patients was 25 months (range, 24.2-25.8). 
Table 1. Baseline characteristics of the patients.

\begin{tabular}{|c|c|c|c|c|c|c|c|c|c|}
\hline & \multicolumn{3}{|c|}{$\begin{array}{l}\text { Patients aged } \\
\geq 60 \text { years }\end{array}$} & \multicolumn{3}{|c|}{$\begin{array}{l}\text { Patients aged } \\
<60 \text { years }\end{array}$} & \multicolumn{3}{|c|}{ 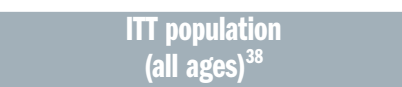 } \\
\hline & $\begin{array}{l}A+A V D \\
(n=84)\end{array}$ & $\begin{array}{c}\text { ABVD } \\
(n=102)\end{array}$ & $\begin{array}{c}\text { Total } \\
(n=186)\end{array}$ & $\begin{array}{c}A+A V D \\
(n=580)\end{array}$ & $\begin{array}{c}\text { ABVD } \\
(n=568)\end{array}$ & $\begin{array}{c}\text { Total } \\
(n=1,148)\end{array}$ & $\begin{array}{l}\text { A+AVD } \\
(n=664)\end{array}$ & $\begin{array}{c}\text { ABVD } \\
(n=670)\end{array}$ & $\begin{array}{c}\text { Total } \\
(\mathrm{N}=1,334)\end{array}$ \\
\hline Median age, & 68 & 66 & 67 & 33 & 33 & 33 & 35 & 37 & 36 \\
\hline years (range) & $(60-82)$ & $(60-83)$ & $(60-83)$ & $(18-59)$ & $(18-59)$ & $(18-59)$ & $(18-82)$ & $(18-83)$ & $(18-83)$ \\
\hline Male, n (\%) & $55(65)$ & $64(63)$ & $119(64)$ & $323(56)$ & $334(59)$ & $657(57)$ & $378(57)$ & $398(59)$ & $776(58)$ \\
\hline White, n (\%) & $76(90)$ & $82(80)$ & $158(85)$ & $484(83)$ & $472(83)$ & $956(83)$ & $560(84)$ & $554(83)$ & $1,114(84)$ \\
\hline \multicolumn{10}{|c|}{ Ann Arbor stage, n (\%)* } \\
\hline III & $31(37)$ & $34(34)$ & $65(35)$ & $206(36)$ & $212(37)$ & $418(36)$ & $237(36)$ & $246(37)$ & $483(36)$ \\
\hline IV & $51(61)$ & $67(66)$ & $118(64)$ & $374(64)$ & $354(62)$ & $728(63)$ & $425(64)$ & $421(63)$ & $846(64)$ \\
\hline \multicolumn{10}{|c|}{ ECOG PS score, n (\%) ${ }^{\dagger}$} \\
\hline 0 & $30(36)$ & $36(36)$ & $66(36)$ & $346(60)$ & $342(60)$ & $688(60)$ & $376(57)$ & $378(57)$ & $754(57)$ \\
\hline 1 & $44(52)$ & $55(54)$ & $99(54)$ & $216(37)$ & $208(37)$ & $424(37)$ & $260(39)$ & $263(39)$ & $523(39)$ \\
\hline 2 & $10(12)$ & $10(10)$ & $20(11)$ & $18(3)$ & $17(3)$ & $35(3)$ & $28(4)$ & $27(4)$ & $55(4)$ \\
\hline
\end{tabular}

A+AVD: brentuximab vedotin plus doxorubicin, vinblastine, and dacarbazine; ABVD: doxorubicin, bleomycin, vinblastine, and dacarbazine; ECOG PS: Eastern Cooperative Oncology Group performance status; ITT: intention-to-treat. *Ann Arbor stage at initial diagnosis was not applicable or missing for four patients; one patient had Ann Arbor stage II disease (major protocol violation). ${ }^{\dagger}$ ECOG PS score was not obtained or missing for two patients.

Modified PFS per IRF was similar in the two treatment arms at 24 months (A+AVD: 70.3\% [95\% CI: 58.4-79.4], ABVD: $71.4 \%$ [95\% CI: 60.5-79.8], $\mathrm{HR}=1.00$ [95\% CI: 0.58-1.72], $P=0.993$ ) (Figure 1A, Table 2). At the end of randomized treatment, the complete response rate per IRF in older patients was $61 \%$ in both arms (difference [A+AVD - ABVD]: -0.1\% [95\% CI: -14.5-14.3]) (Online Supplementary Table S1).

After a median follow-up of 60.9 months' (95\% CI: 60.6-61.7), 5-year PFS per investigator assessment for older cHL patients treated on ECHELON-1 was $67.1 \%$ (95\% CI: 55.1-76.5) with A+AVD versus 61.6\% (95\% CI: 50.9-70.7) with ABVD (HR=0.820 [95\% CI: 0.494-1.362], $P=0.443$ ) (Figure 1B; Table 2). Among younger patients, 5 year PFS per investigator assessment was $84.3 \%$ (95\% CI: 81.0-87.1) and $77.8 \%$ (95\% CI: 74.0-81.1), respectively (HR=0.665 [95\% CI: 0.51-0.88], $P=0.003$ ) (Table 2, Online Supplementary Figure S1).

For older patients, the per investigator PFS was similar in both arms in patients with stage III disease $(\mathrm{HR}=1.051$ [95\% CI: $0.42-2.66], P=0.917)$ or stage IV disease (HR=0.722 [95\% CI: 0.39-1.33], $P=0.291$ ) (Table 2). In exploratory analyses by interim PET scan status after two cycles (PET2), 5-year PFS per investigator assessment for older cHL patients in the A+AVD versus ABVD arm was $71.9 \%$ versus $64.9 \%$ in PET2-negative patients ( $\mathrm{HR}=0.720$ [95\% CI: $0.40-1.29$ ], $P=0.268)$, and $40.0 \%$ versus $25.0 \%$ in PET2-positive patients ( $\mathrm{HR}=0.923$ [95\% CI: 0.23-3.72], $P=0.910)$; however, numbers of patients were low in the PET2-positive, aged $\geq 60$ years subgroup in the $A+A V D$ $\operatorname{arm}(n=5)$ and the ABVD arm $(n=8)$ (Online Supplementary Table S2). For both older and younger cHL patients, PFS rates were higher in PET2-negative versus PET2-positive patients within each study arm (Online Supplementary Table S2).

Per protocol, OS was assessed at the time of the primary analysis (median follow-up 28 months) and the final analysis will be performed once 112 events have occurred in the entire study. Among older patients, 15 patients in the A+AVD arm and 17 in the ABVD arm had died as of the April 20, 2017 data cut. Data on salvage therapy are not available.

\section{Safety}

A total of 181 older patients were evaluable for safety (A+AVD: $n=83$, ABVD: $n=98)$. Older patients received a median of six cycles of treatment across both treatment arms. In the A+AVD arm, $80 \%$ of older patients required one or more dose modification of brentuximab vedotin: dose reduction, $31 \%$; dose held, $5 \%$; dose delayed, $61 \%$; brentuximab vedotin discontinued, $20 \%$. The mean relative dose intensity in older patients for brentuximab vedotin was $92 \%$; relative dose intensities in the A+AVD versus $\mathrm{ABVD}$ arms for doxorubicin were $97 \%$ versus $97 \%$; for vinblastine $93 \%$ versus $93 \%$; and for dacarbazine $98 \%$ versus $96 \%$ (Online Supplementary Table S3). In the ABVD arm, $71 \%$ of older patients required one or more dose modification of bleomycin: dose reduction, $9 \%$; dose held, $4 \%$; dose interrupted, $1 \%$; dose delayed, $49 \%$; bleomycin discontinued, $28 \%$. The mean relative dose intensity for bleomycin was $88.7 \%$ (Online Supplementary Table S3).

Overall, the incidences of grade $\geq 3$ treatment-emergent adverse events were higher in older patients than in younger patients (Table 3 ). Within both age groups, there was a higher incidence of any-grade pulmonary-related events in the ABVD arm than in the A+AVD arm. In older patients, a total of eight deaths occurred on-study (within 30 days of the last dose of frontline treatment), which yielded a treatment-related mortality rate of $4.4 \%(8 / 181$; $3 / 83$ [3.6\%] in the A+AVD arm and 5/98 [5.1\%] in the ABVD arm). Of these eight deaths, three occurred in the A+AVD arm (due to hemophagocytic lymphohistiocytosis, multiple organ dysfunction syndrome, and myocardial infarction [each, $n=1]$ ), none of which was associated with pulmonary toxicity (Online Supplementary Table S4). The remaining five deaths occurred in the ABVD arm (due to pneumonia $[n=2]$, interstitial lung disease $[n=1]$, respiratory disorder $[n=1]$, and cardiac arrest $[n=1])$. Treatment-related pulmonary-related toxicity was associated with three of these five deaths in the ABVD arm, occurring in patients aged 78, 80, and 83 years, and could not be ruled out as having a causal relationship with the other two deaths.

The incidence of grade $\geq 3$ neutropenia was higher in the $\mathrm{A}+\mathrm{AVD}$ arm than in the ABVD arm in older patients $70 \%$ vs. $59 \%$ ). The incidence of any-grade febrile neutropenia 
A

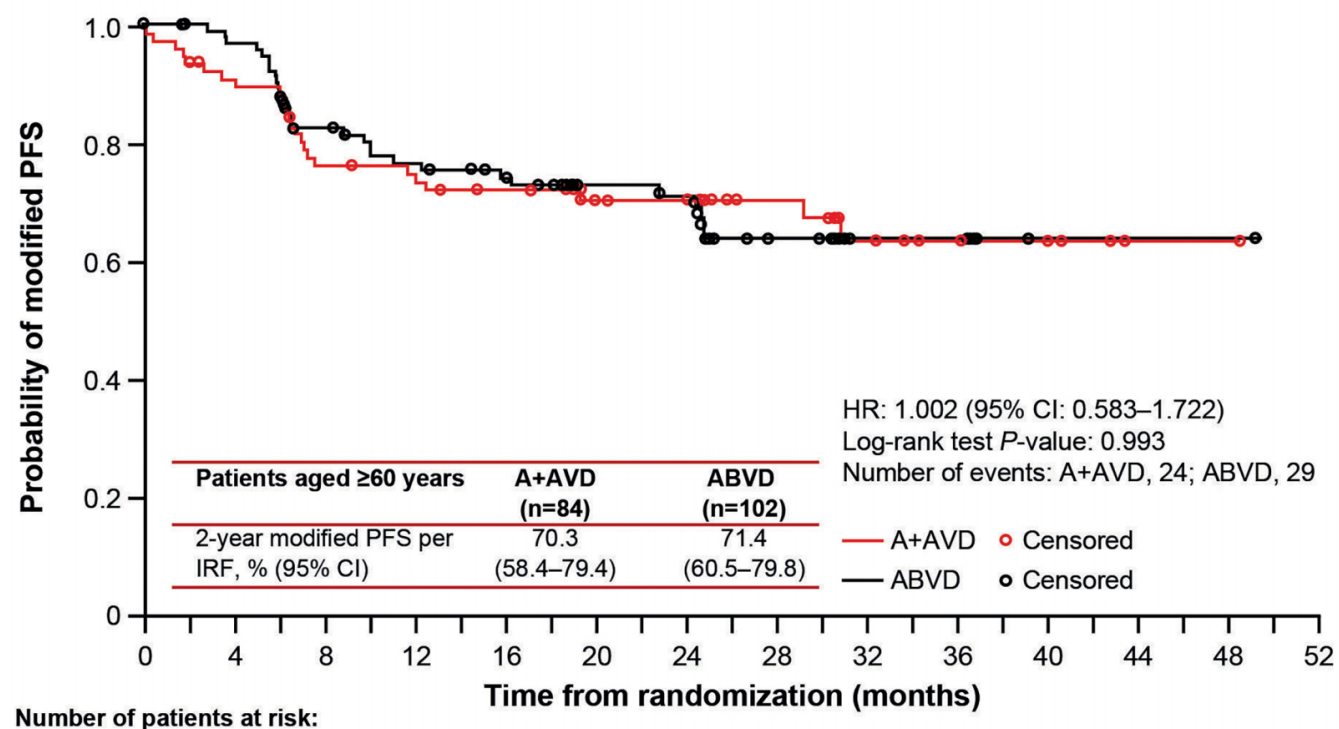

Number of patients at risk:

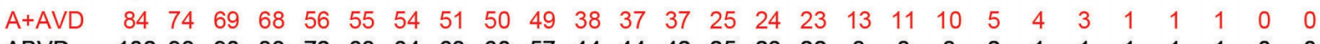

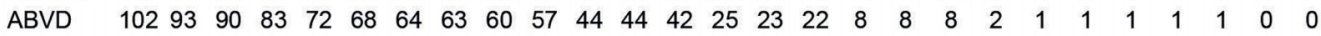

B

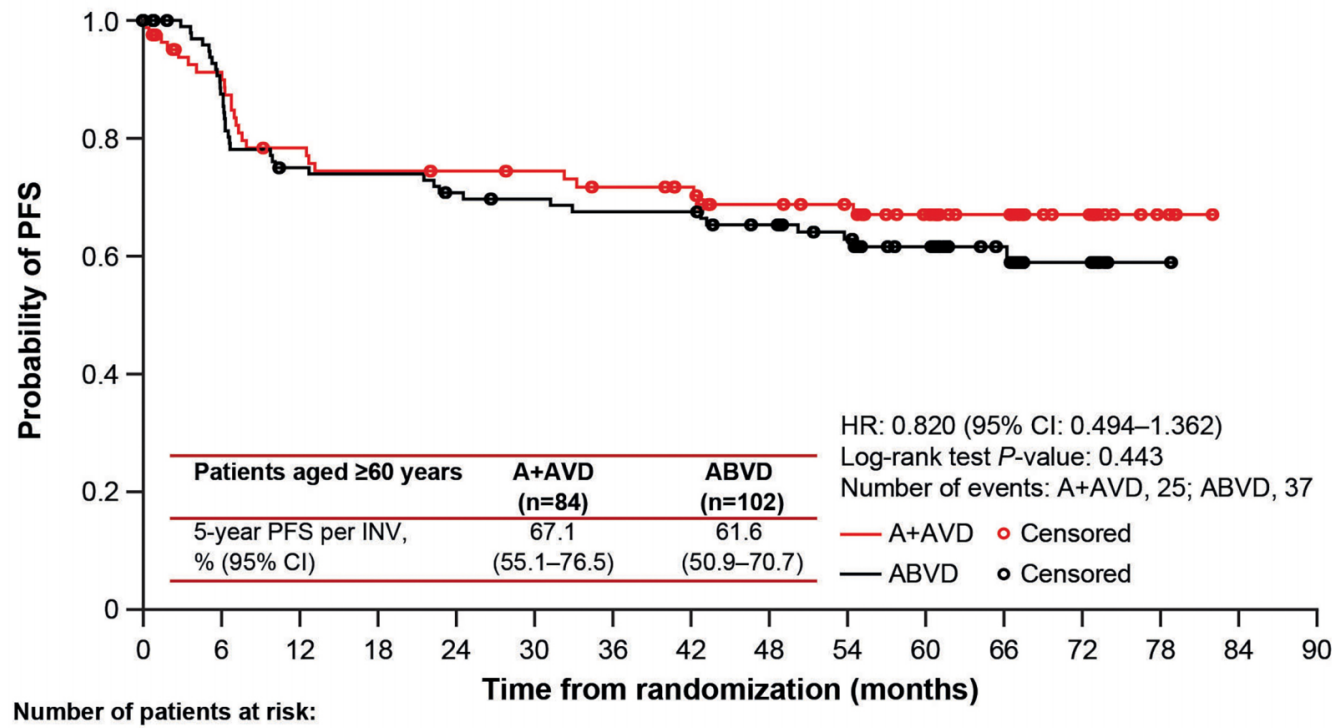

Number of patients at risk:

$\begin{array}{lllllllllllllllll}\text { A+AVD } & 84 & 71 & 60 & 57 & 56 & 55 & 52 & 49 & 43 & 40 & 33 & 23 & 14 & 4 & 0 & 0 \\ & 102 & 84 & 71 & 70 & 66 & 64 & 62 & 62 & 57 & 51 & 42 & 23 & 8 & 1 & 0 & 0\end{array}$

Figure 1. Progression-free survival in patients aged $\geq 60$ years. (A) Modified progression-free survival (PFS) per independent review facility after a median follow-up of 25 months. (B) PFS per investigator after a median follow-up of 60.9 months. A+AVD: brentuximab vedotin plus doxorubicin, vinblastine, and dacarbazine; ABVD: doxorubicin, bleomycin, vinblastine, and dacarbazine; Cl: confidence interval; HR: hazard ratio; INV: investigator; IRF: independent review facility.

was higher in the A+AVD arm than in the ABVD arm in both older patients (37\% vs. 17\%) and younger patients (17\% vs. $6 \%$ ) (Table 3 ). In the A+AVD arm, the use of granulocyte colony-stimulating factor (G-CSF) primary prophylaxis, given per institutional guidelines, was associated with a lower incidence of neutropenia (40\% with $v s$. $78 \%$ without primary prophylaxis) and febrile neutropenia (30\% with vs. $38 \%$ without primary prophylaxis) in older patients (Table 4). The incidence of any-grade peripheral neuropathy was higher in the A+AVD arm than in the ABVD arm in both older (65\% vs. $43 \%)$ and younger patients (67\% vs. $43 \%$ ) (Table 5$)$. Furthermore, the rate of severe grade $3 / 4$ peripheral neuropathy was higher in older patients who received $A+A V D$ than in those who received ABVD (18\% vs. 3\%). Rates of resolution or improvement in peripheral neuropathy appeared similar in older cHL patients treated with A+AVD and ABVD (80\% vs. $83 \%$; respectively). In older patients, 24 and 12 patients had residual peripheral neuropathy, which was grade $1(n=14$ and $n=6)$, grade $2(n=7$ and $n=4)$, and grade $3(n=3$ and $n=2)$ in severity in the $A+A V D$ and ABVD arms, respectively.

\section{Discussion}

Outcomes for older patients with cHL, particularly those with advanced disease, have historically been poor 
Table 2. Summary of modified progression-free survival per independent review facility and per investigator.

\begin{tabular}{|c|c|c|c|c|c|c|c|c|c|c|}
\hline & \multicolumn{2}{|c|}{$\begin{array}{c}\text { Aged } \geq 60 \text { years } \\
(n=186)\end{array}$} & \multicolumn{2}{|c|}{$\begin{array}{l}\text { Aged } \geq 60 \text { years } \\
\text { with stage III } \\
\text { disease }(n=65)^{*}\end{array}$} & \multicolumn{2}{|c|}{$\begin{array}{c}\text { Aged } \geq 60 \text { years } \\
\text { with stage IV } \\
\text { disease }(n=118)^{*}\end{array}$} & \multicolumn{2}{|c|}{$\begin{array}{c}\text { Aged }<60 \text { years } \\
(n=1,148)\end{array}$} & \multicolumn{2}{|c|}{$\begin{array}{c}\text { III population } \\
(n=1,334)\end{array}$} \\
\hline & $\begin{array}{l}A+A V D \\
(n=84)\end{array}$ & $\begin{array}{c}\text { ABVD } \\
(n=102)\end{array}$ & $\begin{array}{l}A+A V D \\
(n=31)\end{array}$ & $\begin{array}{l}\text { ABVD } \\
(n=34)\end{array}$ & $\begin{array}{l}A+A V D \\
(n=51)\end{array}$ & $\begin{array}{l}\text { ABVD } \\
(n=67)\end{array}$ & $\begin{array}{l}A+A V D \\
(n=580)\end{array}$ & $\begin{array}{c}\text { ABVD } \\
(n=568)\end{array}$ & $\begin{array}{l}A+A V D \\
(n=664)\end{array}$ & $\begin{array}{c}\text { ABVD } \\
(n=670)\end{array}$ \\
\hline $\begin{array}{l}\text { 24-month modified } \\
\text { PFS }^{\dagger} \text { per IRF, } \\
\%(95 \% \mathrm{CI})^{20}\end{array}$ & $\begin{array}{c}70.3 \\
(58.4-79.4)\end{array}$ & $\begin{array}{c}71.4 \\
(60.5-79.8)\end{array}$ & $\begin{array}{c}67.7 \\
(44.9-82.6)\end{array}$ & $\begin{array}{c}80.9 \\
(66.2-90.9)\end{array}$ & $\begin{array}{c}71.3 \\
(56.3-81.9)\end{array}$ & $\begin{array}{c}66.1 \\
(51.8-77.1)\end{array}$ & $\begin{array}{c}83.7 \\
(80.2-86.6)\end{array}$ & $\begin{array}{c}78.2 \\
(74.4-81.6)\end{array}$ & $\begin{array}{c}82.1 \\
(78.8-85.0)\end{array}$ & $\begin{array}{c}77.2 \\
(73.7-80.4)\end{array}$ \\
\hline $\begin{array}{l}\text { 24-month } \\
\mathrm{PFS}^{\ddagger} \text { per INV, } \\
\%(95 \% \mathrm{CI})\end{array}$ & $\begin{array}{c}74.4 \\
(62.2-82.7)\end{array}$ & $\begin{array}{c}70.8 \\
(60.6-78.8)\end{array}$ & $\begin{array}{c}74.8 \\
(54.2-87.1)\end{array}$ & $\begin{array}{c}85.3 \\
(68.2-93.6)\end{array}$ & $\begin{array}{c}74.1 \\
(59.6-84.1)\end{array}$ & $\begin{array}{c}62.7 \\
(49.5-73.5)\end{array}$ & $\begin{array}{c}86.5 \\
(83.4-89.1)\end{array}$ & $\begin{array}{c}80.4 \\
(76.8-83.5)\end{array}$ & $\begin{array}{c}84.5 \\
(81.4-87.1)\end{array}$ & $\begin{array}{c}78.3 \\
(74.9-81.4)\end{array}$ \\
\hline $\begin{array}{l}\text { 60-month } \\
\text { PFS }^{\ddagger} \text { per INV, }\end{array}$ & $\begin{array}{c}67.1 \\
(55.1-76.5)\end{array}$ & $\begin{array}{c}61.6 \\
(50.9-70.7)\end{array}$ & $\begin{array}{c}70.1 \\
(48.7-83.9)\end{array}$ & $\begin{array}{c}69.9 \\
(51.3-82.6)\end{array}$ & $\begin{array}{c}65.1 \\
(49.9-76.8)\end{array}$ & $\begin{array}{c}57.0 \\
(43.5-68.5)\end{array}$ & $\begin{array}{c}84.3 \\
(81.0-87.1)\end{array}$ & $\begin{array}{c}77.8 \\
(74.0-81.1)\end{array}$ & $\begin{array}{c}80.7 \\
(77.1-83.8)\end{array}$ & $\begin{array}{c}73.1 \\
(69.0-76.7)\end{array}$ \\
\hline
\end{tabular}

A+AVD: brentuximab vedotin plus doxorubicin, vinblastine, and dacarbazine; ABVD: doxorubicin, bleomycin, vinblastine, and dacarbazine; CI: confidence interval; INV: investigator; IRF: independent review facility; ITT: intention-to-treat; PFS: progression-free survival. *Three patients aged $\geq 60$ years were excluded from analysis by disease stage due to missing data $(\mathrm{n}=2)$ or stage II disease $(\mathrm{n}=1) .{ }^{\dagger} 2$-year modified PFS per IRF based on the primary analysis. ${ }^{\ddagger} 2$ - and 5 -year PFS per INV based on a median of 60.9 months' extended follow-up in patients aged $\geq 60$ years and 60.8 months in patients aged $<60$ years.

Table 3. Safety summary.

\begin{tabular}{|c|c|c|c|c|c|c|}
\hline & $\begin{array}{r}\text { Pati } \\
\geq 60 \text { ye } \\
\text { for saft }\end{array}$ & $\begin{array}{l}\text { aged } \\
\text { evaluable } \\
\text { (n=181) }\end{array}$ & $\begin{array}{l}\text { Pati } \\
<60 \text { yea } \\
\text { for safet }\end{array}$ & $\begin{array}{l}\text { s aged } \\
\text { cvaluable } \\
(n=1,140)\end{array}$ & & $\begin{array}{l}y \\
n^{*, 38}\end{array}$ \\
\hline & $\begin{array}{l}A+A V D \\
(n=83)\end{array}$ & $\begin{array}{l}\text { ABVD } \\
(n=98)\end{array}$ & $\begin{array}{c}\text { A+AVD } \\
(n=579)\end{array}$ & $\begin{array}{c}\text { ABVD } \\
(n=561)\end{array}$ & $\begin{array}{c}A+A V D \\
(n=662)\end{array}$ & $\begin{array}{c}\text { ABVD } \\
(n=659)\end{array}$ \\
\hline Grade $\geq 3 \mathrm{AE}, \mathrm{n}(\%)$ & $73(88)$ & $78(80)$ & $476(82)$ & $356(63)$ & $549(83)$ & $434(66)$ \\
\hline On-study deaths, ${ }^{\dagger} \mathrm{n}(\%)$ & $3(4)$ & $5(5)$ & $6(1)$ & $8(1)$ & $9(1)$ & $13(2)$ \\
\hline Grade $\geq 3$ neutropenia, ${ }^{\ddagger} \mathrm{n}(\%)$ & $58(70)$ & $58(59)$ & $372(64)$ & $259(46)$ & $430(65)$ & $317(48)$ \\
\hline Any-grade FN on study, n (\%) & $31(37)$ & $17(17)$ & $97(17)$ & $35(6)$ & $128(19)$ & $52(8)$ \\
\hline Any-grade pulmonary AE, n (\%) & $2(2)$ & $13(13)$ & $10(2)$ & $31(6)$ & $12(2)$ & $44(7)$ \\
\hline
\end{tabular}

A+AVD: brentuximab vedotin plus doxorubicin, vinblastine, and dacarbazine; ABVD: doxorubicin, bleomycin, vinblastine, and dacarbazine; AE: adverse events; FN: febrile neutropenia. * Received $\geq 1$ dose of study therapy. ${ }^{\dagger}$ Within 30 days of the last dose of frontline treatment. ${ }^{\ddagger}$ Neutropenia includes preferred terms of 'neutropenia' and 'neutrophil count decreased'.

compared with those of younger patients. ${ }^{2-4,6}$ We report here one of the largest prospective, randomized clinical trials in cHL completed in the contemporary era that have included and analyzed the outcomes of older patients. Among older patients $\geq 60$ years treated in ECHELON-1, we report that modified PFS per IRF was statistically similar overall for patients treated with A+AVD or ABVD, being approximately $70 \%$ at 2 years in both arms. After a median follow-up of approximately 5 years, A+AVD demonstrated an apparent treatment benefit, although the numerical improvement in PFS over that with ABVD was not statistically significant. A+AVD was associated with more frequent neuropathy and febrile neutropenia, but less frequent pulmonary toxicity than ABVD. Additionally, older cHL patients had higher rates of febrile neutropenia and neuropathy compared with younger patients treated in ECHELON-1. In interpreting these observations, several factors should be considered.

As older adults may often have multiple comorbidities that pose challenges to the use of traditional multi-agent treatment options, there is a need to identify tolerable and effective treatment regimens. This may reflect improvements in supportive care as well as patient selection. Several recent phase II studies have assessed the efficacy of multiple brentuximab vedotin-based regimens in the frontline cHL setting in older patients. In a phase II study, a sequential administration approach was assessed, in which patients with unfavorable stage II (IIB, IIAX, or IIBX) to stage IV disease received two lead-in doses of single-agent brentuximab vedotin $(1.8 \mathrm{mg} / \mathrm{kg}$ once every 3 weeks), followed by six cycles of AVD. Patients who responded then received four consolidative doses of brentuximab vedotin. ${ }^{19}$ This regimen was well tolerated, with lower rates of grade $\geq 3$ neutropenia $(44 \%)$ and peripheral sensory neuropathy (4\%) compared with those seen in older patients in the A+AVD arm in ECHELON-1, suggesting potentially better tolerability of sequential treatment. ${ }^{9}$ An objective response rate of $95 \%$ (complete responses: $93 \%$ ) and 2-year PFS and OS rates of $84 \%$ and $93 \%$, respectively, were also reported ${ }^{19}$ Furthermore, survival rates varied based on patients' fitness in this study with superior PFS and OS being observed among fit older cHL patients with lower Cumulative Illness Rating ScaleGeriatric co-morbidity scores and those without loss of instrumental activities of daily living, the latter of which persisted on multivariate analyses. Unfortunately, baseline or prospective geriatric assessments were not performed in ECHELON-1.

Brentuximab vedotin has also been assessed as monotherapy and in combination with bendamustine, dacarbazine, or nivolumab. ${ }^{14,16,24,25}$ Importantly, patients enrolled in this study were ineligible for conventional 
Table 4. Safety profile according to receipt of granulocyte colony-stimulating factor primary prophylaxis during days 1-5 of cycle 1.

\begin{tabular}{|c|c|c|c|c|c|c|c|c|}
\hline \multirow[b]{3}{*}{ G-CSF received ${ }^{\dagger}$} & \multicolumn{4}{|c|}{$\begin{array}{l}\text { Patients aged } \geq 60 \text { years } \\
\text { evaluable for safetty* }(n=181)\end{array}$} & \multicolumn{4}{|c|}{$\begin{array}{l}\text { Patients aged }<60 \text { years } \\
\text { evaluable for safety* }(n=1,140)\end{array}$} \\
\hline & \multicolumn{2}{|c|}{$A+A V D(n=83)$} & \multicolumn{2}{|c|}{ ABVD (n=98) } & \multicolumn{2}{|c|}{$A+A V D(n=579)$} & \multicolumn{2}{|c|}{ ABVD (n=561) } \\
\hline & $\begin{array}{c}\text { Yes } \\
(n=10)\end{array}$ & $\underset{(n=73)}{N o}$ & $\begin{array}{c}\text { Yes } \\
(n=9)\end{array}$ & $\underset{(n=89)}{N o}$ & $\begin{array}{c}\text { Yes } \\
(n=73)\end{array}$ & $\underset{(n=506)}{N o}$ & $\begin{array}{c}\text { Yes } \\
(n=34)\end{array}$ & $\begin{array}{c}\text { No } \\
(n=527)\end{array}$ \\
\hline Any-grade neutropenia, n (\%) & $4(40)$ & $57(78)$ & $1(11)$ & $64(72)$ & $25(34)$ & 368 (73) & $8(24)$ & $288(55)$ \\
\hline FN in cycle $1, \mathrm{n}(\%)$ & $1(10)$ & $20(27)$ & $2(22)$ & $8(9)$ & 0 & $41(8)$ & 0 & $16(3)$ \\
\hline Any-grade FN on study, n (\%) & $3(30)$ & $28(38)$ & $2(22)$ & $15(17)$ & $6(8)$ & $91(18)$ & $1(3)$ & $34(6)$ \\
\hline $\begin{array}{l}\text { Infections and Infestations } \\
\text { System Organ Class, n (\%) }\end{array}$ & $8(80)$ & $43(59)$ & $5(56)$ & $60(67)$ & $31(42)$ & $279(55)$ & $14(41)$ & $252(48)$ \\
\hline Any SAE on study, n (\%) & $5(50)$ & $53(73)$ & $2(22)$ & $44(49)$ & $22(30)$ & $204(40)$ & $5(15)$ & $127(24)$ \\
\hline
\end{tabular}

A+AVD: brentuximab vedotin plus doxorubicin, vinblastine, and dacarbazine; ABVD: doxorubicin, bleomycin, vinblastine, and dacarbazine; FN: febrile neutropenia; G-CSF: granulocyte colony-stimulating factor; SAE: serious adverse event. ${ }^{*}$ Received $\geq 1$ dose of study therapy. ${ }^{\dagger} \mathrm{G}$-CSF was given per institutional practice.

Table 5. Peripheral neuropathy: incidence and resolution.

\begin{tabular}{|c|c|c|c|c|}
\hline & \multicolumn{2}{|c|}{$\begin{array}{l}\text { Patients aged } \geq 60 \text { years } \\
\text { evaluable for safety* } \\
\qquad(n=181)\end{array}$} & \multicolumn{2}{|c|}{$\begin{array}{l}\text { Patients aged }<60 \text { years } \\
\text { evaluable for safety* } \\
\qquad(n=1,140)\end{array}$} \\
\hline & $\begin{array}{l}A+A V D \\
(n=83)\end{array}$ & $\begin{array}{c}A B V D \\
(n=98)\end{array}$ & $\begin{array}{c}A+A V D \\
(n=579)\end{array}$ & $\begin{array}{c}\text { ABVD } \\
(n=561)\end{array}$ \\
\hline Any-grade PN, n/N (\%) & $54 / 83(65)$ & $42 / 98(43)$ & $389 / 579(67)$ & $244 / 561(43)$ \\
\hline Grade 1 PN, n/N (\%) & 23/83 (28) & $26 / 98$ (27) & 219/579 (38) & 192/561 (34) \\
\hline Grade 2 PN, n/N (\%) & $16 / 83(19)$ & $13 / 98(13)$ & $114 / 579(20)$ & $44 / 561(8)$ \\
\hline Grade 3/4 PN, ${ }^{\dagger}$ n/N (\%) & 15/83 (18) & 3/98 (3) & $56 / 579(9)$ & $8 / 561(1)$ \\
\hline $\begin{array}{l}\text { Patients with PN and complete } \\
\text { resolution/improvement, n/N (\%) }\end{array}$ & $43 / 54(80)$ & $35 / 42(83)$ & $332 / 389(85)$ & $210 / 244(86)$ \\
\hline PN complete resolution, $\mathrm{n} / \mathrm{N}(\%)$ & $30 / 54(56)$ & $30 / 42(71)$ & 286/389 (74) & $197 / 244(81)$ \\
\hline PN improvement, n/N (\%) & $13 / 54(24)$ & $5 / 42(12)$ & $46 / 389(12)$ & $13 / 244(5)$ \\
\hline
\end{tabular}

A+AVD: brentuximab vedotin plus doxorubicin, vinblastine, and dacarbazine;ABVD: doxorubicin, bleomycin, vinblastine, and dacarbazine; PN: peripheral neuropathy. ${ }^{\text {Received }}$ $\geq 1$ dose of study therapy. ${ }^{\dagger}$ Among all patients evaluable for safety $(n=1,321)$, only one case of grade 4 PN was reported, and this event occurred in a patient aged $<60$ years in the A+AVD arm.

frontline chemotherapy combinations (according to the investigator's judgement). Initial assessment of brentuximab vedotin monotherapy demonstrated promising efficacy with $92 \%$ of patients (median age, 78 years) achieving an objective response (complete response rate: $73 \%)^{14}$ The combinations of brentuximab vedotin with dacarbazine, bendamustine, or nivolumab produced 100\% objective response rates with each regimen (complete response rates: $62 \%, 88 \%$, and $72 \%$, respectively). ${ }^{16,26}$ Enrollment to the bendamustine combination was discontinued because of $65 \%$ of patients experiencing serious adverse events. ${ }^{16}$ Updated analyses with median followups of 59.4 and 58.6 months in the brentuximab vedotin monotherapy and dacarbazine and nivolumab combination therapy arms, respectively, showed median PFS of 10.5 and 46.8 months, and OS of 77.5 and 64.0 months, respectively. The median PFS and OS had not been reached in the nivolumab arm, with a median follow-up of 19.4 months. ${ }^{25}$ The authors concluded that brentuximab vedotin plus dacarbazine or nivolumab were reasonable combinations in this more unfit/frail population of patients. The nivolumab combination was associated with a higher rate of grade $\geq 3$ treatment-related adverse events compared with the dacarbazine combination $(60 \%$ vs. $37 \%$ ), including peripheral neuropathy ( $35 \%$ vs. $26 \%$ ), but a lower rate of serious treatment-related adverse events $(5 \%$ vs. $11 \%)$ and treatment discontinuations due to adverse events (30\% vs. $42 \%)$. ${ }^{25}$ Another phase II study of brentuximab vedotin plus nivolumab in previously untreated older patients ( $\geq 60$ years) suggested a lower objective response rate of $64 \%$, including $52 \%$ with complete responses, at an interim analysis, which indicated that the combination was active in this population but did not meet predefined criteria that required a higher level of activity for further enrollment in the trial to proceed. ${ }^{27}$

The overall incidence of treatment-emergent adverse events in ECHELON-1 was comparable in the A+AVD and ABVD arms. A lower incidence of pulmonary-related toxicity was observed in the A+AVD arm than in the ABVD arm for both older and younger patients, with this difference being more marked in older patients. In older patients, three out of five on-study deaths in the ABVD arm were associated with pulmonary toxicity compared with none in the A+AVD arm (Online Supplementary Table S4), with $28 \%$ of patients in the ABVD treatment arm requiring bleomycin discontinuation. Since this study was initiated, it has been shown that pulmonary toxicity with $A B V D$ can be reduced without reducing efficacy by omitting bleomycin from the regimen after two cycles in PET2-negative patients. ${ }^{28}$ The decision over whether to use this risk-adapted approach over $\mathrm{A}+\mathrm{AVD}$ requires an assessment of the efficacy benefits, safety, and treatment costs for each individual patient. For older patients, the increased risk of toxicity, during the first two cycles of 
treatment in those receiving PET2-adapted therapy and for PET2-positive patients (who continue on more intensive therapy), must be considered. However, treatment intensification is not recommended for older patients because of poor tolerance of BEACOPP. ${ }^{29}$ In the randomized $\mathrm{HD} 9_{\text {elderly }}$ study comparing baseline-BEACOPP regimen with cyclophosphamide, vincristine, procarbazine, prednisone + ABVD (COPP-ABVD), the treatment-related mortality rates among 75 patients with advanced-stage HL aged $66-75$ years were $21 \%$ and $8 \%$, respectively. ${ }^{7} \mathrm{~A}$ modified regimen incorporating brentuximab vedotin, dacarbazine, and dexamethasone (BrECADD) in place of bleomycin, vincristine, procarbazine, and prednisone (as used in BEACOPP) is being investigated in a phase III trial (HD21; NCT02661503) ${ }^{30}$ after a phase II study found that this regimen was associated with a relatively favorable toxicity profile while maintaining a complete response rate of $88 \%$. $^{31}$

Microtubule inhibitors, such as the vinca alkaloids (e.g., vinblastine and vincristine) and the monomethyl auristatin $\mathrm{E}$ component of brentuximab vedotin are associated with occurrence of peripheral neuropathy. ${ }^{32-34}$ The incidence of any-grade peripheral neuropathy in older patients was higher with A+AVD than with ABVD $(65 \%$ vs. $43 \%)$, especially grade $3 / 4$ peripheral neuropathy $(18 \%$ vs. $3 \%$ ). Severe peripheral neuropathy was also more frequently seen in older than younger $\mathrm{cHL}$ patients treated with $A+A V D$. In the $A+A V D$ arm, approximately fourfifths of older patients with peripheral neuropathy experienced improvement or resolution, a rate similar to that observed in the ABVD arm. With longer follow-up, residual peripheral neuropathy continues to improve and resolve. ${ }^{21,35}$ These findings highlight the importance of appropriate screening, monitoring, and active clinical management of peripheral neuropathy in patients treated with A+AVD (including potential dose reductions particularly in older patients who frequently present with multiple comorbidities).

In the current analyses, the rates of neutropenia and febrile neutropenia were higher in the A+AVD arm overall and, moreover, higher in older than younger patients in both the A+AVD and ABVD arms. Although the use of primary prophylaxis with G-CSF was not mandated in ECHELON-1 and the cohort of older patients who received G-CSF primary prophylaxis was small $(n=10)$, post-protocol amendment use of G-CSF primary prophylaxis was associated with reduced rates of neutropenia and febrile neutropenia in patients treated with A+AVD. Similar effects of primary prophylaxis with G-CSF on rates of neutropenia and febrile neutropenia were observed in patients treated with $A+A V D$ in the overall ECHELON-1 study population. ${ }^{36}$ Consequently, G-CSF primary prophylaxis is recommended for all patients who receive $A+A V D .{ }^{37}$ As the optimal dosing schedule has not been established, G-CSF should be administered with each cycle, starting at cycle 1 , as recommended in the US prescribing information and EU Summary of Product Characteristics.

Taken together, these data showed overall similar efficacy for $\mathrm{A}+\mathrm{AVD}$ and $\mathrm{ABVD}$ in older patients with stage III/IV cHL. A+AVD was associated with increased neuropathy and neutropenia but with less pulmonary-related toxicity compared with ABVD. Thus, A+AVD represents a treatment option (with primary prophylaxis with G-CSF) for selected fit, older patients with cHL overall, and especially for patients in whom pulmonary toxicity is a concern. Moreover, outcomes reported here set a new benchmark for older patients with untreated $\mathrm{cHL}$ when treated with $A+A V D$ or ABVD. However, continued study of new therapeutic regimens is needed to improve outcomes and to decrease toxicity for older cHL patients. This includes continued examination of PET responseadapted strategies, which may be prognostic in brentuximab vedotin-based treatment for older cHL patients, ${ }^{19,21}$ as well as analysis of timing of brentuximab vedotin relative to chemotherapy (i.e., sequential vs. concurrent), integration of other targeted therapeutic agents (e.g., NCT03907488), and via the incorporation of objective geriatric assessments for prediction of tolerable and individualized therapy.

\section{Disclosures}

AME reports consultancy with honoraria for MorphoSys, Miltenyi, Seagen, and Epizyme; membership on an advisory committee with honoraria for Pharmacyclics and Novartis, Inc.; and research funding from Tesaro. JMC reports research funding from Merck, Amgen, Roche Canada, NanoString Technologies, Seagen, Janssen, F. Hoffmann-La Roche, Bayer Healthcare, Cephalon, Bristol Myers-Squibb, Lilly, Genentech, and Millennium Pharmaceuticals Inc., a wholly owned subsidiary of Takeda Pharmaceutical Company Limited; being a named inventor on a patent licensed to NanoString Technologies; and honoraria from Seagen. AY reports research funding from J\&J, Bristol Myers-Squibb, Curis, Genentech, Pharmacyclics, Janssen, Novartis, Roche, Abbvie, and Astra Zeneca; and honoraria from Celgene, Bristol Myers-Squibb, Sanofi, Abbvie, Merck, Bayer, Incyte, Seagen, Roche, and Takeda. SMA reports research funding from $L A M$ Therapeutics, Regeneron, Pfizer, Bristol-Myers Squibb, Merck \& Co, Trillium, Seagen, Celldex, Takeda, and Affimed. JR reports research funding from Celgene, ADC Therapeutics, Pfizer, and Millennium Pharmaceuticals Inc., a wholly owned subsidiary of Takeda Pharmaceutical Company Limited; consultancy for ADC Therapeutics, Novartis, Takeda, Seagen, and Bristol Myers-Squibb; equity ownership in GlaxoSmithKline and AstraZeneca; and speakers bureau for Novartis, Takeda, Seagen, and Bristol Myers-Squibb. TF reports research funding from Seagen and Portola; and speakers bureau for Seagen, Janssen, Pharmacyclics, JQJ, Celgene, and KITE. JT reports research funding from Millennium Pharmaceuticals Inc., a wholly owned subsidiary of Takeda Pharmaceutical Company Limited, Genentech, Celgene, and Pharmacyclics; speakers bureau for Amgen, Seagen, and Celgene; and honoraria from Amgen, Seagen, and Celgene. KJS reports honoraria from and consulting for Seagen, Merck, Bristol Myers-Squibb, Abbvie, Astra Zeneca, Verastem, and Gilead Consulting Servier; and research funding from Seagen, Millennium Pharmaceuticals Inc., a wholly owned subsidiary of Takeda Pharmaceutical Company, Merck, Bristol MyersSquibb, Beigene, and Roche Canada. YO reports employment with Genentech and research funding from Seagen and Millennium Pharmaceuticals Inc., a wholly owned subsidiary of Takeda Pharmaceutical Company Limited; honoraria from Takeda Millennium; and employment with Jazz Pharmaceuticals. AGri reports membership on an entity's Board of Directors or advisory committees for Roche, Gilead, Bristol Myers-Squibb, and Takeda. CP reports employment with Kent \& Canterbury Hospital. MD-D reports consultancy for Servier and Roche. KF reports employment with and equity ownership in Seagen, Inc. AF-T reports employment with Seagen, Inc. RL, HJ, and AGau report employment with Millennium Pharmaceuticals, 
Inc., a wholly owned subsidiary of Takeda Pharmaceutical Company Limited. AGal reports consultancy and speakers bureau for Takeda. W-SK has declared no conflicts of interest.

\section{Contributions}

Data were verified by the sponsor, analyzed by sponsor statisticians, and interpreted by academic authors and sponsor representatives. The manuscript was prepared by the authors with the assistance of a medical writer funded by the sponsor. Data were collected, and study procedures were overseen by $A M E, J M C, A Y$, SMA, WSK, JR, TF, JT, KJS, YO, AG, CP, MD-D, and AGal; data were verified by KF, $A F-T, R L, H T$, and $A G$ al, analyzed by $R L$, and interpreted by all authors. All authors had full access to the data during development of the manuscript. All authors vouch for completeness and accuracy of the data and had final responsibility for the manuscript content and decision to submit.

\section{Acknowledgments}

The authors would like to thank the patients who participated in this study and their families. They would also like to acknowledge other investigators and staff at all ECHELON-1 clinical sites and the members of the Independent Data Monitoring Committee and Independent Review Committee. The authors acknowledge the writing assistance of Laura Webb and Hedley Coppock of Ashfield MedComms, an Ashfield Health company, part of UDG Healthcare plc, during the development of this manuscript, which was funded by Millennium Pharmaceuticals, Inc., and complied with the Good Publication Practice 3 ethical guidelines. ${ }^{39}$

\section{Funding}

This work was supported by Millennium Pharmaceuticals, Inc., Cambridge, MA, USA, a wholly owned subsidiary of Takeda Pharmaceutical Company Limited (grant number not applicable); and Seagen, Inc., Bothell, WA, USA (grant number not applicable).

\section{Data-sharing statement}

The datasets, including the redacted study protocol, redacted statistical analysis plan, and individual participant's data supporting the results reported in this article, will be made available within 3 months from initial request, to researchers who provide a methodologically sound proposal. The data will be provided after de-identification, in compliance with applicable privacy laws, data protection, and requirements for consent and anonymization.

\section{References}

1. Thyss A, Saada E, Gastaud L, Peyrade F, Re D. Hodgkin's lymphoma in older patients: an orphan disease? Mediterr J Hematol Infect Dis. 2014;6(1):e2014050.

2. Proctor SJ, Wilkinson J, Jones G, et al Evaluation of treatment outcome in 175 patients with Hodgkin lymphoma aged 60 years or over: the SHIELD study. Blood. 2012;119(25):6005-6015.

3. Stark GL, Wood KM, Jack F, et al. Hodgkin's disease in the elderly: a population-based study. Br J Haematol. 2002;119(2):432-440.

4. Engert A, Ballova V, Haverkamp H, et al. Hodgkin's lymphoma in elderly patients: a comprehensive retrospective analysis from the German Hodgkin's Study Group. J Clin Oncol. 2005;23(22):5052-5060.

5. Evens AM, Hong F, Gordon LI, et al. The efficacy and tolerability of adriamycin, bleomycin, vinblastine, dacarbazine and Stanford V in older Hodgkin lymphoma patients: a comprehensive analysis from the North American intergroup trial E2496. Br J Haematol. 2013;161(1):76-86

6. Evens AM, Hong F. How can outcomes be improved for older patients with Hodgkin lymphoma? J Clin Oncol. 2013;31(12):15021505.

7. Ballova V, Ruffer JU, Haverkamp H, et al. A prospectively randomized trial carried out by the German Hodgkin Study Group (GHSG) for elderly patients with advanced Hodgkin's disease comparing BEACOPP baseline and COPP-ABVD (study HD9elderly). Ann Oncol. 2005;16(1):124-131.

8. Evens AM, Helenowski I, Ramsdale E, et al. A retrospective multicenter analysis of elderly Hodgkin lymphoma: outcomes and prognostic factors in the modern era. Blood. 2012;119(3):692-695.

9. Boll B, Goergen $\mathrm{H}$, Behringer $\mathrm{K}$, et al. Bleomycin in older early-stage favorable Hodgkin lymphoma patients: analysis of the German Hodgkin Study Group (GHSG) HD10 and HD13 trials. Blood. 2016; 127(18):2189-2192.
10. Stamatoullas A, Brice P, Bouabdallah R, et al Outcome of patients older than 60 years with classical Hodgkin lymphoma treated with front line ABVD chemotherapy: frequent pulmonary events suggest limiting the use of bleomycin in the elderly. Br J Haematol. 2015;170(2):179-184.

11. Sleijfer S. Bleomycin-induced pneumonitis Chest. 2001;120(2):617-624.

12. Martin WG, Ristow KM, Habermann TM, et al. Bleomycin pulmonary toxicity has a negative impact on the outcome of patients with Hodgkin's lymphoma. J Clin Oncol. 2005;23(30):7614-7620.

13. Thomas TS, Luo S, Reagan PM, et al. Advancing age and the risk of bleomycin pulmonary toxicity in a largely older cohort of patients with newly diagnosed Hodgkin lymphoma. J Geriatr Oncol. 2020;11(1):69-74.

14. Forero-Torres A, Holkova B, Goldschmidt J, et al. Phase 2 study of frontline brentuximab vedotin monotherapy in Hodgkin lymphoma patients aged 60 years and older. Blood. 2015;126(26):2798-2804

15. Gibb A, Pirrie SJ, Linton K, et al. Results of a UK National Cancer Research Institute phase II study of brentuximab vedotin using a response-adapted design in the first-line treatment of patients with classical Hodgkin lymphoma unsuitable for chemotherapy due to age, frailty or comorbidity (BREVITY). Br J Haematol. 2020;193(1):63-71.

16. Friedberg JW, Forero-Torres A, Bordoni RE, et al. Frontline brentuximab vedotin in combination with dacarbazine or bendamustine in patients aged $\geq 60$ years with HL. Blood. 2017;130(26):2829-2837.

17. Gallamini A, Bijou F, Viotti J, et al Brentuximab-vedotin and bendamustine is a feasible and effective drug combination as first-line treatment of Hodgkin lymphoma in the elderly (HALO trial). Hematol Oncol. 2017;35(Suppl 2):170

18. Fosså A, Böll B, Goergen $\mathrm{H}$, et al. T021 (0147) B-CAP (brentuximab vedotin cyclophosphamide, doxorubicin and predniso(lo) $\mathrm{Ne}$ ) in older patients with advancedstage Hodgkin lymphoma: results of a phase
II intergroup trial by the German Hodgkin Study Group (GHSG) and the Nordic Lymphoma Group (NLG). HemaSphere. 2018;2:27

19. Evens AM, Advani RH, Helenowski IB, et al Multicenter phase II study of sequentia brentuximab vedotin and doxorubicin, vinblastine, and dacarbazine chemotherapy for older patients with untreated classical Hodgkin lymphoma. J Clin Oncol. 2018; 36(30):3015-3022

20. Connors JM, Jurczak W, Straus DJ, et al. Brentuximab vedotin with chemotherapy for stage III or IV Hodgkin's lymphoma. N Engl J Med. 2018;378(4):331-344.

21. Straus DJ, Diugosz-Danecka M, Alekseev S, et al. Brentuximab vedotin with chemotherapy for stage III/IV classical Hodgkin lymphoma: 3-year update of the ECHELON-1 study. Blood. 2020;135(10):735-742

22. Straus DJ, Długosz-Danecka M, Connors JM, et al. Brentuximab vedotin with chemotherapy for stage III or IV classical Hodgkin lymphoma (ECHELON-1): 5-year update of an international, open-label, randomised, phase 3 trial. Lancet Haematol. 2021;8(6):e410-e421.

23. Cheson BD, Pfistner B, Juweid ME, et al. Revised response criteria for malignant lymphoma. J Clin Oncol. 2007;25(5):579-586.

24. Friedberg JW, Forero-Torres A, Holkova B, et al. Long-term follow-up of brentuximab vedotin \pm dacarbazine as first line therapy in elderly patients with Hodgkin lymphoma. J Clin Oncol. 2018;36(Suppl 15):7542

25. Yasenchak CA, Bordoni R, Patel-Donnelly $\mathrm{D}$, et al. Frontline brentuximab vedotin as monotherapy or in combination for older Hodgkin lymphoma patients. Blood. 2020 136(Suppl 1):18-19.

26. Yasenchak CA, Bordoni R, Yazbeck V, et al. Phase 2 study of frontline brentuximab vedotin plus nivolumab in patients with Hodgkin lymphoma aged $\geq 60$ years. Blood. 2019;134(Suppl 1):237.

27. Cheson $\mathrm{BD}$, Bartlett NL, LaPlant $\mathrm{B}$, et al. Brentuximab vedotin plus nivolumab as first-line therapy in older or chemotherapy- 
ineligible patients with Hodgkin lymphoma (ACCRU): a multicentre, single-arm, phase 2 trial. Lancet Haematol. 2020;7(11):e808e815.

28. Johnson P, Federico M, Kirkwood A, et al. Adapted treatment guided by interim PETCT scan in advanced Hodgkin's lymphoma. N Engl J Med. 2016;374(25):2419-2429.

29. Spinner MA, Advani RH. Risk-adapted therapy for advanced-stage Hodgkin lymphoma. Hematology Am Soc Hematol Educ Program. 2018;2018(1):200-206.

30. ClinicalTrials.gov. HD21 for Advanced Stages. NCT02661503. [last accessed May 18, 2021]; Available from: https:// clinicaltrials.gov/ct2/show/NCT02661503?term $=\mathrm{HD}$ 21\&cond=Hodgkin + Lymphoma\&draw $=2 \&$ rank=1.

31. Eichenauer DA, Plütschow A, Kreissl S, et al. Incorporation of brentuximab vedotin into first-line treatment of advanced classical
Hodgkin's lymphoma: final analysis of a phase 2 randomised trial by the German Hodgkin Study Group. Lancet Oncol. 2017; 18(12):1680-1687.

32. van de Donk NW, Dhimolea E. Brentuximab vedotin. MAbs. 2012;4(4):458-465.

33. Donaghy $\mathrm{H}$. Effects of antibody, drug and linker on the preclinical and clinical toxicities of antibody-drug conjugates. MAbs. 2016; 8(4):659-671.

34. Addington J, Freimer M. Chemotherapyinduced peripheral neuropathy: an update on the current understanding. F1000Res. 2016;5:F1000 Faculty Rev-1466.

35. Radford J, Connors JM, Younes A, et al Resolution of peripheral neuropathy $(\mathrm{PN})$ in patients who received $A+A V D$ or $A B V D$ in the phase 3 ECHELON-1 trial. Blood. 2018; 132(Suppl 1):2921.

36. Straus D, Collins G, Walewski J, et al. Primary prophylaxis with G-CSF may improve outcomes in patients with newly diagnosed stage III/IV Hodgkin lymphoma treated with brentuximab vedotin plus chemotherapy. Leuk Lymphoma. 2020; 61(12):2931-2938.

37. Sureda A, Domingo-Domenech E, Gautam A. Neutropenia during frontline treatment of advanced Hodgkin lymphoma: incidence, risk factors, and management. Crit Rev Oncol Hematol. 2019;138:1-5.

38. Straus DJ, Collins GP, Walewski JA, et al. Improving outcomes with brentuximab vedotin (BV) plus chemotherapy in patients with newly diagnosed advanced stage Hodgkin lymphoma. J Clin Oncol. 2018; 36(Suppl 15):7534.

39. Battisti WP, Wager E, Baltzer L, et al. Good Publication practice for communicating company-sponsored medical research GPP3. Ann Intern Med. 2015;163(6):461464. 